\title{
Tunneling of electrons through semiconductor superlattices
}

\author{
C L ROY \\ Department of Physics and Meteorology, Indian Institute of Technology, Kharagpur 721 302, India
}

\begin{abstract}
The purpose of the present paper is to report a study of tunneling of electrons through semiconductor superlattices (SSL); specially, we have analysed diverse features of transmission coefficient of SSL. The SSL we have considered is $\mathrm{Ga}_{0.7} \mathrm{Al}_{0.3} \mathrm{As}-\mathrm{GaAs}$ which has been drawing considerable attention during the recent past on account of some typical features of its band structure. We have indicated how our results would help fabrication of ultra high speed devices.
\end{abstract}

Keywords. Semiconductor superlattices; tunneling.

\section{Introduction}

Semiconductor superlattices (SSL) are fabricated by growing two different semiconducting compounds in alternate layers, generally with thickness two to twenty times the lattice spacing. Semiconductor superlattices provide systems which are useful in regard to (i) investigation of some typical features of electrical conduction in presence of moderate fields and (ii) high speed devices (Mendez et al 1988; Voisin et al 1988; Mahapatra et al 1998). A SSL which has received considerable attention is the $\mathrm{Ga}_{0.7} \mathrm{Al}_{0.3} \mathrm{As}-\mathrm{GaAs}$ system. In the present paper, we report a study of tunneling of electrons through this SSL. For the purpose, we have obtained the transmission coefficient for a very general form of SSL and carried out quantitative analysis of diverse features of tunneling through the above mentioned SSL with special attention to resonant tunneling (RT) which plays an important role for high speed devices.

\section{The model}

The model we have treated is shown in figure $1 \mathrm{~b}$, this model is a multibarrier system (MBS) which is a generalization of the one shown in figure 1a (Esaki 1986). Figure 1a shows that the potential $\mathrm{W}$ in a superlattice with components $\mathrm{A}$ and $\mathrm{B}$ is taken to be alternately rectangular barriers and wells at the conduction and valence band edges along the growth direction. The material A with lower band gap $\left(E_{\mathrm{g} 2}\right)$ is flanked by the material $\mathrm{B}$ with higher band gap $\left(E_{\mathrm{g} 1}\right)$. The model of figure $1 \mathrm{~b}$ is a repetition of wells and barriers of figure 1a, subject to the following conditions: (i) the barrier height $V=\left(E_{\mathrm{g} 1}-E_{\mathrm{g} 2}\right)$ and (ii) the masses of the electron in the well and barrier regions are different and they correspond respectively to the effective masses at the conduction band edges of $\mathrm{A}$ and $\mathrm{B}$.
As shown in figure 1a, the SSL we would treat quantitatively is the one with $\mathrm{A}$ as $\mathrm{Ga}_{0.7} \mathrm{Al}_{0.3} \mathrm{As}$ and $\mathrm{B}$ as GaAs.

\section{Transmission coefficient and related issues}

\subsection{Boundary conditions}

The BC for the wave function $(\psi)$ at points of potential discontinuity like $\mathrm{P}$ in figure 1a are: (i) continuity of $\psi$ and (ii) continuity of $\frac{1}{m}\left(\frac{\mathrm{d} \psi}{\mathrm{d} x}\right)$, where ' $m$ ' is the mass of the electron at $x$. The BC (i) and (ii) are necessary for keeping the probability density $(\rho)$ and the current density $\left(J_{x}\right)$ continuous where

$$
\begin{aligned}
& \rho=\psi^{*} \psi \\
& J_{x}=-\frac{e \hbar}{2 m c}\left(\psi^{*} \frac{\mathrm{d} \psi}{\mathrm{d} x}-\psi \frac{\mathrm{d} \psi^{*}}{\mathrm{~d} x}\right) .
\end{aligned}
$$

\subsection{Transmission coefficient}

We assumed a beam of electrons to be incident at the left end of the MBS in figure $1 \mathrm{~b}$ and allow them to get transmitted to the right of the MBS. At the left end, there will be a right-going current density $\left(J_{\mathrm{i}}\right)$ and a left-going (reflected) current density $\left(J_{\mathrm{r}}\right)$. At the right end of the MBS, there is only a right-going (transmitted) current density $\left(J_{\mathrm{t}}\right)$. The TC $\left(C_{\mathrm{N}}\right)$ for the MBS is defined as

$$
C_{\mathrm{N}}=\frac{J_{\mathrm{t}}}{J_{\mathrm{i}}}
$$

Applying the BC mentioned in 3.1 to the solutions of Schrodinger equation for various regions of the MBS, we obtain $C_{\mathrm{N}}$ as shown below

$$
C_{\mathrm{N}}=C_{\mathrm{N}}^{\mathrm{I}}=\left[1+|D|^{2}\left(U_{\mathrm{r}}(\cos \psi)\right)^{2}\right]^{-1},|g|<2,
$$



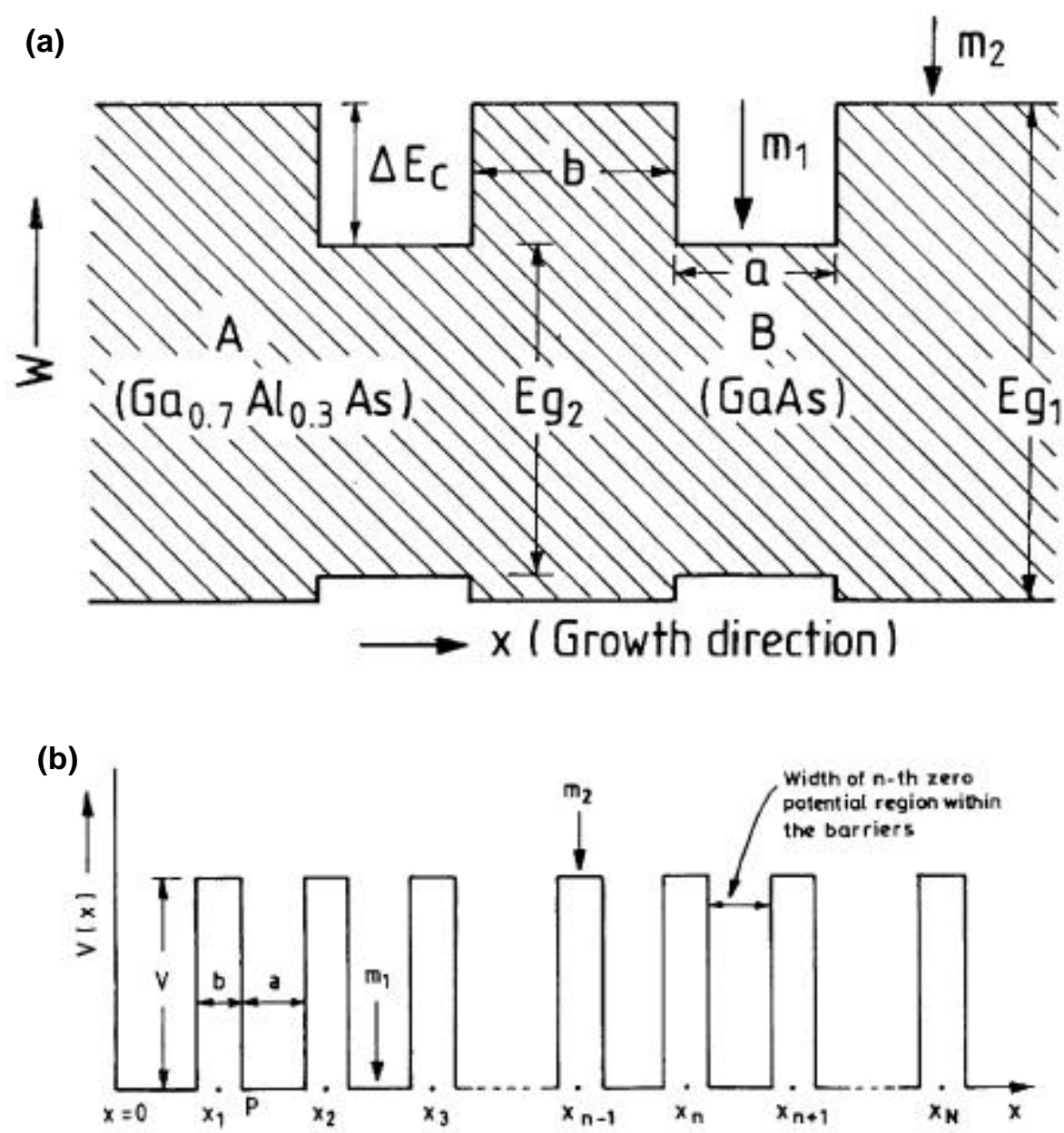

Figure 1. a. Periodic potential in a semiconductor superlattice. The symbols are explained in the text and $\mathbf{b}$. multibarrier system with repetition of wells and barriers of figure 1a.

$C_{\mathrm{N}}=C_{\mathrm{N}}^{\mathrm{II}}=\left[1+|D|^{2}\left(H_{\mathrm{r}}(\cos \eta)\right)^{2}\right]^{-1},|g| \geq 2$,

$U_{\mathrm{r}}(\cos \psi)=\frac{\sin (r+1) \psi}{\sin \psi}$,

$H_{\mathrm{r}}(\cos \eta)=\frac{\sin h(r+1) \eta}{\sin h \eta}$,

$r=(N-1) ; \quad \cos \psi=\frac{g}{2},|g|<2 ; \quad \cos h \eta=\frac{g}{2},|g| \geq 2$,

$g=2\left[\cos Q_{1} a \cosh \rho Q_{2} b+\left(\rho^{2} Q_{2}^{2}-Q_{1}^{2}\right) \sin Q_{1}\right.$ $\left.a \sin h\left(\rho Q_{2} b\right)\left(2 \rho Q_{1} Q_{2}\right)^{-1}\right]$,

$D=-i\left[Q_{2}^{1}+\rho^{2} Q_{2}^{2}\right]\left[\sin h\left(\rho Q_{2} b\right)\right]\left[2 \rho Q_{1} Q_{2}\right]^{-4}$,

$Q_{1}^{2}=\frac{2 m_{1} E}{h^{2}} ; \quad Q_{2}^{2}=\frac{2 m_{2}(V-E)}{h^{2}} ; \rho=\frac{m_{1}}{m_{2}}, E<V$
The well known resonant tunneling (RT) corresponds to $C_{\mathrm{N}}=1$; we can easily see that RT can occur at energies which are under the control of $C_{\mathrm{N}}^{\mathrm{I}}$.

\section{Quantitative results}

We have shown quantitatively how $C_{\mathrm{N}}(E)$ varies with $E$ for the $\mathrm{Ga}_{0.7} \mathrm{Al}_{0.3} \mathrm{As}-\mathrm{GaAs}$ superlattice. For this SSL, $m_{1}=0.067 \mathrm{~m}, m_{2}=0.092 \mathrm{~m}, V=0.33 \mathrm{eV} ; m$ is the free mass of the electron. The results are shown in figures $2 b$ and $3 \mathrm{~b}$; for comparison, we have shown the results of figures $2 \mathrm{a}$ and $3 \mathrm{a}$.

\section{Conclusions}

The major conclusions arising out of our results are as follows:

(I) For the SSL we have treated, the number of RT peaks 

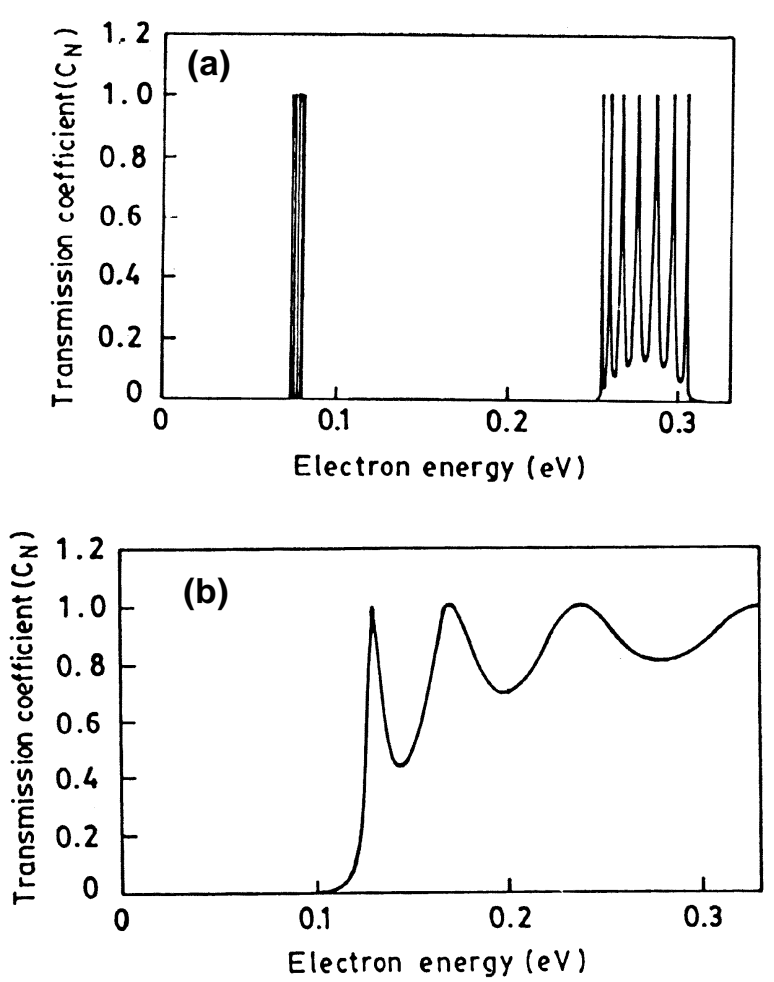

Figure 2. a. Transmission coefficient for multibarrier system $\left(N=8, V=0.33 \mathrm{eV}, m_{1}=m_{2}=m\right)$ and $\mathbf{b}$. transmission coefficient for the superlattice, $\mathrm{Ga}_{0.7} \mathrm{Al}_{0.3} \mathrm{As}-\mathrm{GaAs}(N=8, V=0.33 \mathrm{eV}$, $\left.m_{1}=0.067 \mathrm{~m}, m_{2}=0.092 \mathrm{~m}\right)$.

increases generally with $N$ and at the same time, such peaks become shaper for larger $N$.

(II) Other parameters being same, the TC with $m_{1} \neq m_{2}$ differs substantially from that with $m_{1}=m_{2}$, in respect of (i) the number of RT peaks and (ii) the sharpness of RT peaks.

(III) Features such as those in (I) and (II) play an important role in regard to effective fabrication of high speed devices.

\section{Acknowledgements}

The author is grateful to Prof. H N Acharya and Prof.
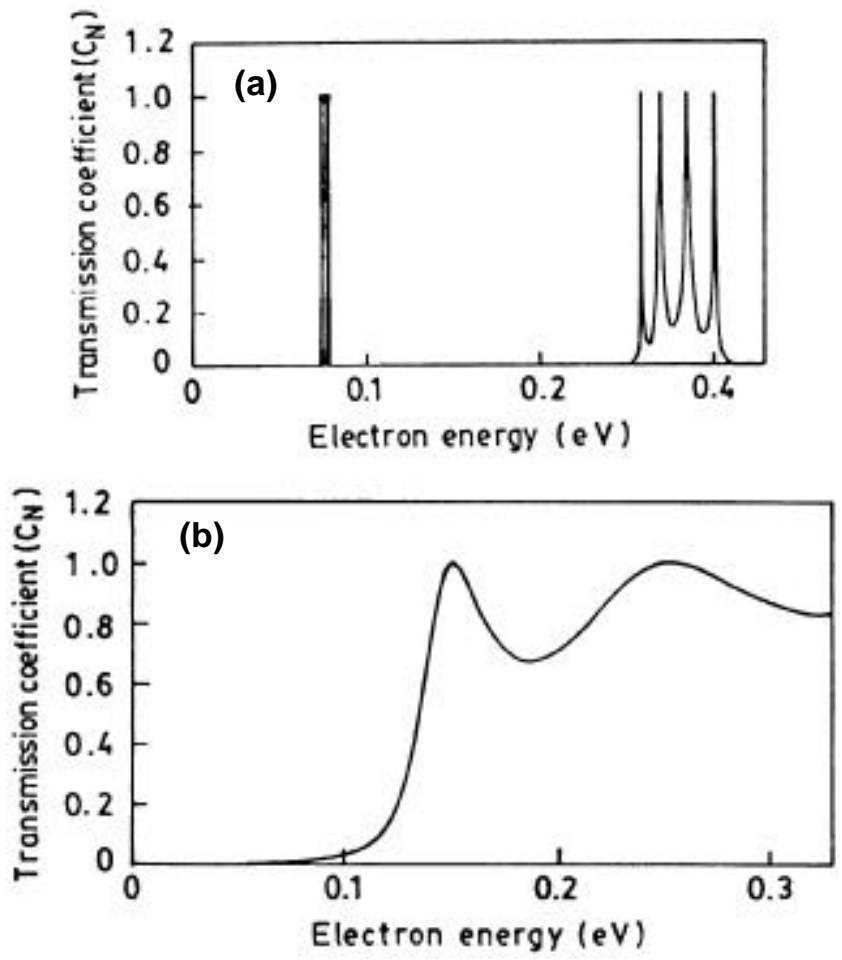

Figure 3. a. The same kind of graph as in figure $2 \mathrm{a}$ for $N=5$; other parameters are the same as in figure $2 \mathbf{a}$ and $\mathbf{b}$. the same kind of graph as in figure $2 \mathrm{~b}$ for $N=5$; other parameters are the same as in figure $2 b$.

R N P Choudhury, Department of Physics and Meteorology, IIT, Kharagpur, for facilities.

\section{References}

Esaki L 1986 IEEE J. Electron. 221611

Mahapatra P K, Bhattacharya K, Khan A and Roy C L 1998 Phys. Rev. $\mathrm{B58} 1560$

Mendez E E, Agullo-Rueda F and Hong J M 1988 Phys. Rev. Lett. 602426

Voisin P, Bleuse J, Bouche C, Gaillard S, Albert C and Regency A 1988 Phys. Rev. Lett. 611639 